Research article

\title{
Taxonomical and phytosociological studies on Chithalikavu- A sacred grove, Thrissur district, Kerala
}

\author{
Deepa M. R. ${ }^{1 *}$, P. S. Udayan ${ }^{1}$ and Anilkumar K. A. ${ }^{2}$ \\ ${ }^{1}$ P.G. and Research Department of Botany, Sree Krishna College, Guruvayur, Ariyannur, \\ Thrissur-680102, Kerala, India \\ ${ }^{2}$ Spices Board, Ministry of Commerce and Industry, Government of India, West Sikkim-737111, India
}

*Corresponding Author: deepakrishna56@gmail.com

[Accepted: 15 January 2017]

\begin{abstract}
Groves contains natural forest and also rich in biodiversity. They are protected by local communities because of their deities linked with these forest patches. The present study was conducted in Chithalikavu, a sacred grove of Thrissur district to know the plant diversity and their structural parameters. Floristic composition of Chithalikavu sacred grove revealed that the occurrence of 57 species of angiosperms belonged to 54 genus and 35 families. Among them $29.82 \%$ trees, $24.56 \%$ shrubs, $15.79 \%$ herbs and $29.83 \%$ climbers. The parameters like frequency, relative frequency, density, relative density and importance value index were estimated by using standard procedures. Strychnos nux-vomica was recorded as the most dominant species in the community as it constituted highest IVI. Other dominant species of the community were Ficus benghalensis and Terminalia paniculata. The highest basal area was reported in Ficus benghalensis.
\end{abstract}

Keywords: Chithalikavu - Sacred grove - Kerala - Phytosociology - Quadrets - Taboos.

[Cite as: Deepa MR, Udayan PS \& Anilkumar KA (2017) Taxonomical and phytosociological studies on Chithalikavu- A sacred grove, Thrissur district, Kerala. Tropical Plant Research 4(1): 20-30]

\section{INTRODUCTION}

Sacred groves are small patches of native forests and act as abodes of gods, protected by local communities, exist all over the world. These are known as "Kavu" or "Sarpakavu" in Kerala. According to Malhotra et al. (2001) groves are those area dedicated by local communities to their ancestral spirits or deities. These have immense value from genetic diversity as well as ecological point of view and rich in flora. They are the repository of several medicinal and economically important plants. Attached with socio-cultural and religious sentiments these exist as undisturbed islands. But today these are adversely affected by human activities.

These sacred groves are protected usually through taboos and sanctions with cultural and ecological implications. There are more than 2000 sacred groves occur in Kerala (Pushpangadan et al. 1998). These acts as treasure houses of plants and animals and can satisfy scientific, cultural and aesthetic needs of mankind. In Kerala groves are mainly dedicated to Serpant gods and Folk deities. Many threatened species existed in the sacred groves of Kerala (Nair \& Mohanan 1981) and it preserving unique species of plants, insects and animals (Venkatachalam et al. 2005). Therefore it acts as a tool for biodiversity conservation (Gaikwad et al. 2004).

\section{MATERIALS AND METHODS}

Study Area

The study was conducted in Chithalikavu, a sacred grove of Talappilly taluk, Thrissur district, Kerala state, lies in $10^{\circ} 73^{\prime} 21^{\prime \prime} \mathrm{N}$ and $76^{\circ} 42^{\prime} 73^{\prime \prime} \mathrm{E}$ with an area of $c a .1 .73$ ha. The management of these kavu is under the control of Chithali family members. The main deities are Nagam (Snake God) and Bhagavathy (Godes). Protection of this kavu is mainly due to the presence of deities. This area receives both Southwest and Northeast monsoons during June-August and October-December respectively. In Chithalikavu compound wall or fencing were absent, therefore external disturbance like grazing, stem cutting, illegal medicinal plants collection are present and one side of grove soil had less humus cover. 


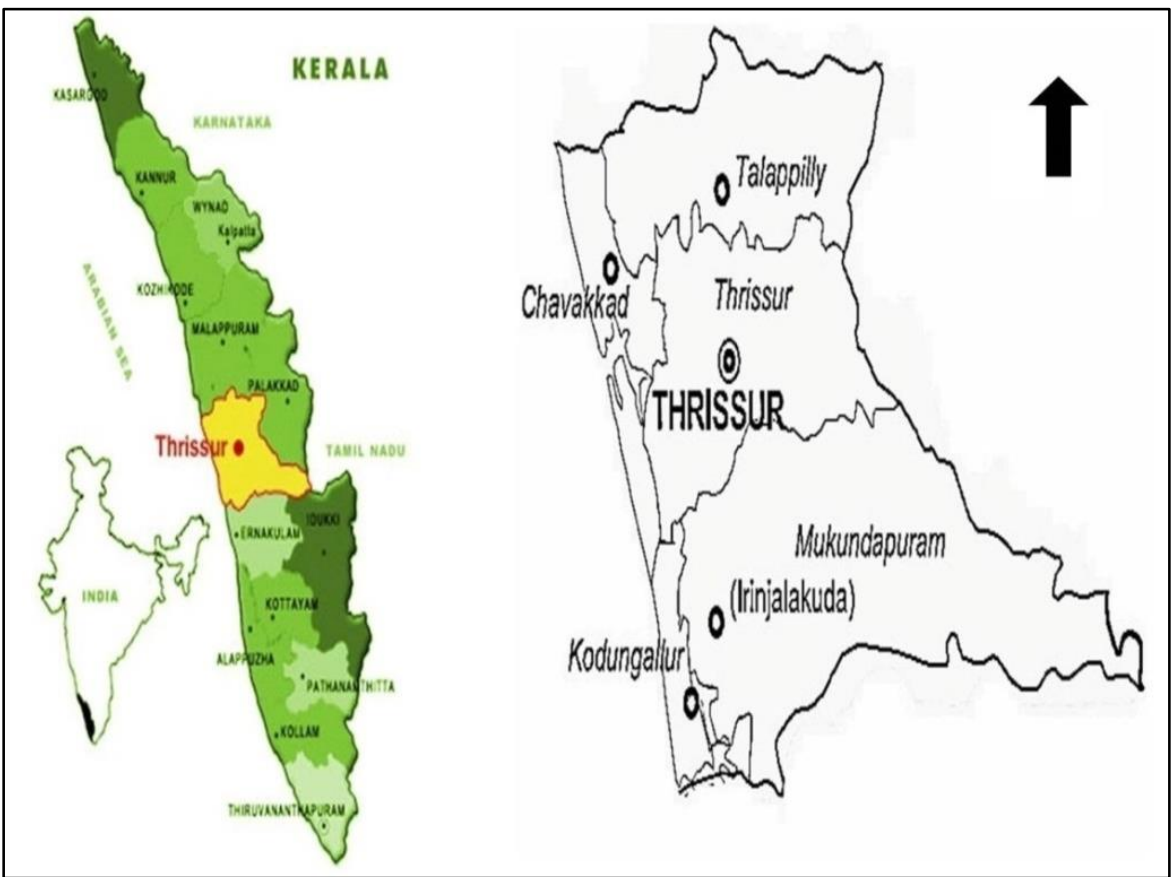

Figure 1. Map showing the study area: Thrissur district of Kerala, India. [Adopted from Deepa et al. (2016)]

\section{Plant collection}

Floristic composition of this grove was recorded during field visits and plant collection conducted over different seasons between April 2013 and September 2015. All the Angiosperms including trees, shrubs, herbs and climbers were considered for the study. Important field observation such as habit, habitat, local names and medicinal uses available were noted in the field book. Plant materials of proper size with relevant parts were collected from the field and sealed in polyethylene covers after treating with formaldehyde. Herbaria were prepared, processed and labeled by following standard herbarium methods given by Jain \& Rao (1977). Each species in fresh condition were critically studied with the help of floras (Gamble 1915-1936, Sasidharan \& Sivarajan 1996), Monographs, publications, etc and provisional determination was made. The identity of the taxon was confirmed with type materials deposited at E, K, CAL, MH, KFRI and protologue. The voucher specimens are deposited at Sree Krishna College, Guruvayur, Thrissur, Kerala.

\section{Ecological data collection and analysis}

Phytosociological study by quadrate method was also conducted for describing the Structural features of the grove. Size and number of the quadrate was determined by the species area curve method (Shailaja \& Sudha 2001). Two quadrates of $20 \mathrm{~m} \times 20 \mathrm{~m}$ size were randomly established in the study site for the studies of tree species (Bajpai et al. 2015). Each quadrate was then systematically surveyed by identifying and all trees with girth at breast height (gbh) greater than and equal to $30 \mathrm{~cm}$ were recorded for analysis. Tree girth measurements were made as per Poffenberger et al. (1992).

The numerical value obtained were analyzed to find out frequency, relative frequency, density, relative density, relative basal area and importance value index were estimated by using standard formula (Curtis \& McIntosh 1950, Krebs 1989, Phillips 1959).

\section{RESULTS}

The present taxonomic study in Chithalikavu resulted in the collection and identification of 57 species of angiosperms belonged to 54 genus and 35 families. Among them $29.82 \%$ trees, $24.56 \%$ shrubs, $15.79 \%$ herbs and $29.82 \%$ climbers. During the study an endemic plant to Peninsular India (Canthium rheedei DC.), and under two near threatened species like Piper longum L. and Tinospora sinensis (Lour.) Merr. (Ravikumar \& Ved 2000) were collected. Almost all the plants found in the grove have medicinal properties.

During this study Euphorbiaceae was recorded as the dominant family with seven species of six genera, followed by Fabaceae (five species of five genera) while Amaranthaceae, Meliaceae, Poaceae, Oxalidaceae, Bombacaceae, Caricaceae, Combretaceae, Convolvulaceae, Cucurbitaceae, Dioscoreaceae, Hypoxidaceae, Leeaceae, Liliaceae, Loganiaceae, Oleaceae, Periplocaceae, Piperaceae, Poaceae, Ranunculaceae are the 
families represented by only one species. Phyllanthus, Sida and Tinospora were the largest genus with 2 species while 51 genera were denoted by only one species in each. Because of their medicinal values Aerva lanata (L.) Juss. ex Schult., Azadirachta indica A. Juss., Canthium rheedei DC., Cassia fistula L., Ficus benghalensis L., Gloriosa superba L., Glycosmis pentaphylla (Retz.) DC., Naravelia zeylanica (L.) DC., Piper longum L., Strychnos nux-vomica L., Terminalia paniculata Roth. and Tinospora cordifolia (WIlld.) Miers. are the some important plants of the study area (Fig. 2). Botanical name of each plant with their family, local name, habit phenology (flowering and fruiting) and medicinal uses have been provided in table 1.
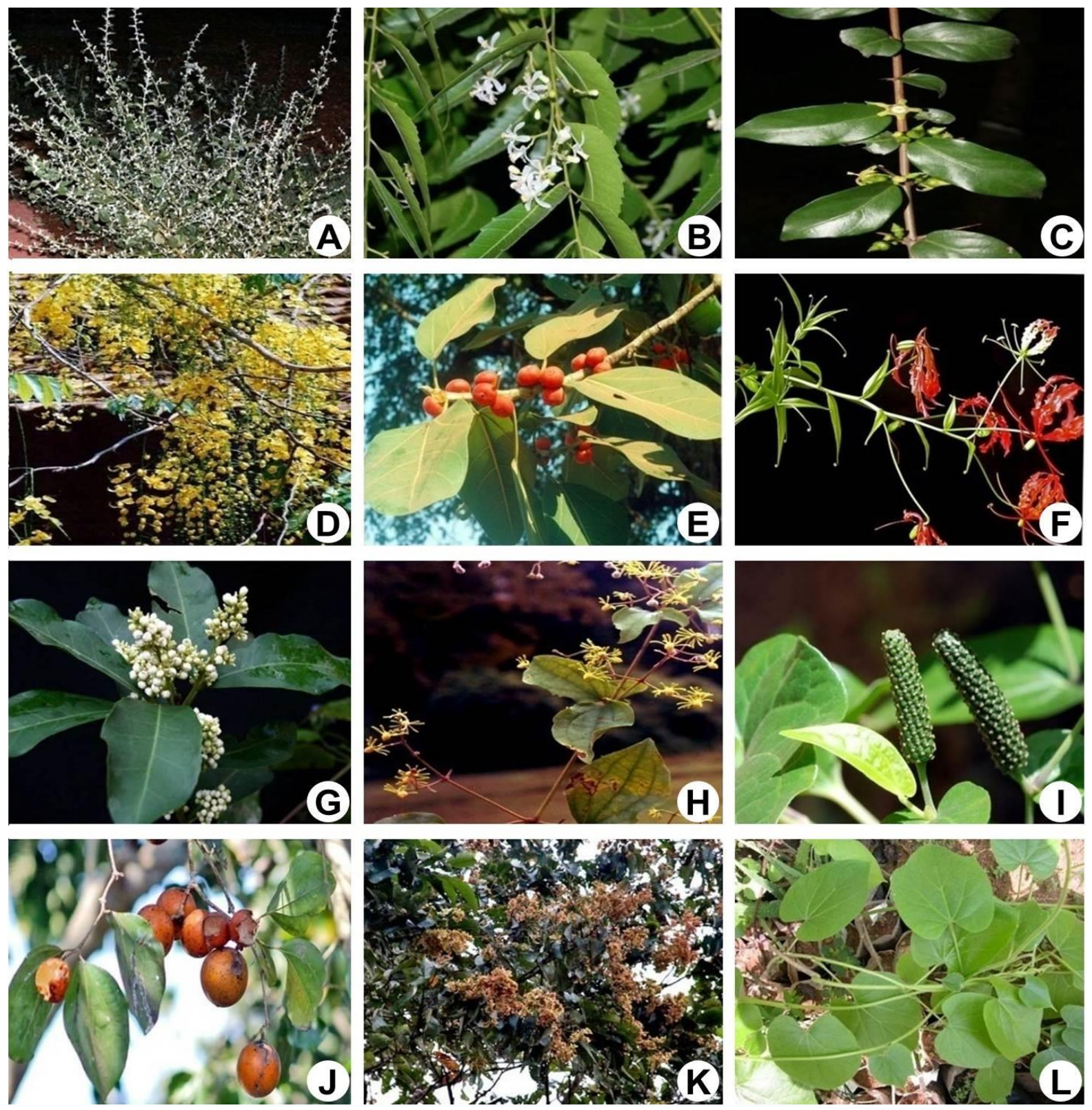

Figure 2. Some important plants in Chithalikavu: A, Aerva lanata (L.) Juss. ex Schult.; B, Azadirachta indica A. Juss.; C, Canthium rheedei DC.; D, Cassia fistula L.; E, Ficus benghalensis L.; F, Gloriosa superba L.; G, Glycosmis pentaphylla (Retz.) DC.; H, Naravelia zeylanica (L.) DC.; I, Piper longum L.; J, Strychnos nux-vomica L.; K, Terminalia paniculata Roth.; L, Tinospora cordifolia (WIlld.) Miers.

Only eight tree species were observed in the study area. Among them Strychnos nux-vomica L. was the dominating species because of having the maximum value of Importance Value Index (IVI) Beside Strychnos nux-vomica (IVI = 1.085), Ficus benghalensis L. (0.634), Terminalia paniculata Roth (0.338) and Schleichera oleosa (Lour.) Oken (0.309) were the other important tree species from Chithalikavu. Maximum density was showed by Strychnos nux-vomica as $325 \mathrm{ha}^{-1}$ followed by Caryota urens L. $\left(37.5 \mathrm{ha}^{-1}\right)$ and Terminalia paniculata Roth $\left(25 \mathrm{ha}^{-1}\right)$ (Table 2). The highest relative basal area was recorded in Ficus benghalensis L. (0.515). 
Table 1. Species recorded from sacred groves with their family, local name, habit, phenology (flowering and fruiting) and medicinal uses. (H- Herb, S- Shrub, T- Tree, C- Climber)

\begin{tabular}{|c|c|c|c|c|c|c|c|}
\hline $\begin{array}{l}\text { S. } \\
\text { No. }\end{array}$ & $\begin{array}{l}\text { Col. } \\
\text { No. }\end{array}$ & Botanical name & Family & Local name & Habit & Phenology & Medicinal uses \\
\hline 1 & 198 & $\begin{array}{l}\text { Aerva lanata (L.) Juss. ex } \\
\text { Schult. }\end{array}$ & Amaranthaceae & Cherula & $\mathrm{H}$ & $\begin{array}{l}\text { September - } \\
\text { April }\end{array}$ & $\begin{array}{l}\text { Used in urinary obstructions, } \\
\text { bladder stones and haemorrhages } \\
\text { associated with pregnancy. }\end{array}$ \\
\hline 2 & 061 & $\begin{array}{l}\text { Albizia saman (Jacq.) F. } \\
\text { Muell. }\end{array}$ & Mimosaceae & Mazhamaram & $\mathrm{T}$ & $\begin{array}{l}\text { March - } \\
\text { May }\end{array}$ & $\begin{array}{l}\text { Used in hot baths for stomach } \\
\text { cancer and remedy for colds, } \\
\text { diarrhea, headache, intestinal } \\
\text { ailments, stomachache and sore } \\
\text { throat. }\end{array}$ \\
\hline 3 & 014 & $\begin{array}{l}\text { Amorphophallus } \\
\text { paeoniifolius (Dennst) } \\
\text { Nicolson }\end{array}$ & Araceae & Kattuchena & $\mathrm{H}$ & $\begin{array}{l}\text { May - } \\
\text { June }\end{array}$ & $\begin{array}{l}\text { Used in dysentery, piles, cough, } \\
\text { applied externally to treat } \\
\text { rheumatism, opthalmia, cure } \\
\text { diarrhea, rheumatic swellings, } \\
\text { elephantiasis and vomiting. }\end{array}$ \\
\hline 4 & 114 & $\begin{array}{l}\text { Azadirachta indica A. } \\
\text { Juss. }\end{array}$ & Meliaceae & Ariyavepu & $\mathrm{T}$ & $\begin{array}{l}\text { February - } \\
\text { September }\end{array}$ & $\begin{array}{l}\text { Used in skin diseases, ulcers, } \\
\text { eczema, rheumatism, intestinal } \\
\text { worms, impurity of blood, eye } \\
\text { diseases, diabetes, small pox, } \\
\text { chiken pox, ringworm, scabies etc. }\end{array}$ \\
\hline 5 & 144 & $\begin{array}{l}\text { Bambusa bambos (L.) } \\
\text { Voss }\end{array}$ & Poaceae & Mula & $\mathrm{S}$ & $\begin{array}{l}\text { July - } \\
\text { February }\end{array}$ & $\begin{array}{l}\text { Haemorrhoid, diarrhoea, wounds, } \\
\text { skin diseases, fever, cough, } \\
\text { shortness of breath, vomiting, } \\
\text { cardiac diseases and skin diseases }\end{array}$ \\
\hline 6 & 146 & $\begin{array}{l}\text { Biophytum reinwardtii } \\
\text { (Zucc.) Klotzsch }\end{array}$ & Oxalidaceae & Mukkutti & $\mathrm{H}$ & $\begin{array}{l}\text { July - } \\
\text { December }\end{array}$ & $\begin{array}{l}\text { It is used in strangury, urninary } \\
\text { calculi, hyperdipsia, wounds, } \\
\text { asthma, stomachalgia, snakebite } \\
\text { and insomnia. }\end{array}$ \\
\hline 7 & 070 & Bombax ceiba L. & Bombacaceae & Poola & $\mathrm{T}$ & $\begin{array}{l}\text { January - } \\
\text { April }\end{array}$ & $\begin{array}{l}\text { Used in calculous affections and } \\
\text { ulceration of bladder and kidneys, } \\
\text { dysentery, pulmonary tuberculosis, } \\
\text { influenza, menorrhagia, fever, } \\
\text { burning sensation and skin } \\
\text { eruptions. }\end{array}$ \\
\hline 8 & 073 & Canthium rheedei DC. & Rubiaceae & Edalimaram & $\mathrm{S}$ & $\begin{array}{l}\text { March - } \\
\text { June }\end{array}$ & $\begin{array}{l}\text { Usedfor whitish ulcers on the } \\
\text { surface of a mucous membrane, } \\
\text { better for obstructions of the liver, } \\
\text { purifies, blood and cheers up the } \\
\text { patient. }\end{array}$ \\
\hline 9 & 058 & Capsicum frutescens $\mathrm{L}$. & Solanaceae & $\begin{array}{l}\text { Kantharimul- } \\
\text { aku }\end{array}$ & $\mathrm{H}$ & $\begin{array}{l}\text { Throughout } \\
\text { the year }\end{array}$ & $\begin{array}{l}\text { Used as carminative and } \\
\text { rubefacient. }\end{array}$ \\
\hline 10 & 274 & $\begin{array}{l}\text { Cardiospermum } \\
\text { helicacabum } \mathrm{L} .\end{array}$ & Sapindaceae & Uzhinja & $\mathrm{C}$ & $\begin{array}{l}\text { July - } \\
\text { February }\end{array}$ & $\begin{array}{l}\text { Whole plant is used for hair growth, } \\
\text { rheumatism, glandular swellings, } \\
\text { constipation, nervous disorders, } \\
\text { piles, chronic bronchitis, fever, } \\
\text { hydrocele, sprains and cardiopathy. }\end{array}$ \\
\hline
\end{tabular}




\begin{tabular}{|c|c|c|c|c|c|c|c|}
\hline 11 & 148 & Carica papaya $\mathrm{L}$. & Caricaceae & Pappaya & $\mathrm{S}$ & $\begin{array}{l}\text { Throughout } \\
\text { the year }\end{array}$ & $\begin{array}{l}\text { Fruits, seed, leaf and latex used in } \\
\text { hemiplegia, rheumatoid arthritis, } \\
\text { anorexia, indigestion, sprue, colic, } \\
\text { stomachalgia, dyspepsia, intestinal } \\
\text { worms, inflammations, piles, } \\
\text { cardiac diseases, oedema, fever, } \\
\text { ringworm, skin diseases and } \\
\text { leprosy. }\end{array}$ \\
\hline 12 & 006 & Caryota urens L. & Arecaceae & Aanapana & $\mathrm{T}$ & $\begin{array}{l}\text { January - } \\
\text { April }\end{array}$ & $\begin{array}{l}\text { The leaf bud, seed and toddy are } \\
\text { used for diarrhoea, migraine and } \\
\text { scorpion-sting poisoning. }\end{array}$ \\
\hline 13 & 149 & Cassia fistula $\mathrm{L}$. & Caesalpiniaceae & Konna & $\mathrm{T}$ & $\begin{array}{l}\text { February - } \\
\text { September }\end{array}$ & $\begin{array}{l}\text { Remedy for skin diseases, leprosy, } \\
\text { fever, promotes digestion, } \\
\text { leucoderma, eczema, diabetes, } \\
\text { cardiac diseases, jaundice, polyuria, } \\
\text { and urticaria. }\end{array}$ \\
\hline 14 & 150 & $\begin{array}{l}\text { Cayratia pedata (Lam.) } \\
\text { A. Juss. ex Gagnep. }\end{array}$ & Vitaceae & $\begin{array}{l}\text { Veluthasori- } \\
\text { valli }\end{array}$ & $\mathrm{C}$ & $\begin{array}{l}\text { June - } \\
\text { July }\end{array}$ & $\begin{array}{l}\text { Leaf decoction is used to check } \\
\text { uterine reflexes. Roots made into a } \\
\text { paste and slightly heated are } \\
\text { applied on cracked heels. }\end{array}$ \\
\hline 15 & 115 & $\begin{array}{l}\text { Chromolaena odorata } \\
\text { (L.) King \& Robins. }\end{array}$ & Asteraceae & $\begin{array}{l}\text { Communist- } \\
\text { pacha }\end{array}$ & $\mathrm{S}$ & $\begin{array}{l}\text { November - } \\
\text { May }\end{array}$ & $\begin{array}{l}\text { Leaf juice is applied externally on } \\
\text { cuts and wounds to stop bleeding. }\end{array}$ \\
\hline 16 & 016 & Cissus latifolia Lam. & Vitaceae & $\begin{array}{l}\text { Chunnambu- } \\
\text { valli }\end{array}$ & $\mathrm{C}$ & $\begin{array}{l}\text { June - } \\
\text { September }\end{array}$ & $\begin{array}{l}\text { Used for the treatment of burning } \\
\text { fever, cough, purifies blood, cure } \\
\text { the ulcer of lungs. }\end{array}$ \\
\hline 17 & 306 & $\begin{array}{l}\text { Cleistanthus collinus } \\
\text { (Roxb.) Benth. ex Hook. f. }\end{array}$ & Euphorbiaceae & Odugu & $\mathrm{T}$ & $\begin{array}{l}\text { December - } \\
\text { November }\end{array}$ & $\begin{array}{l}\text { Leaves, roots and fruits act as } \\
\text { gastrointestinal irritant. Fruits used } \\
\text { for treating cancer. }\end{array}$ \\
\hline 18 & 054 & $\begin{array}{l}\text { Curculigo orchioides } \\
\text { Gaertn. }\end{array}$ & Hypoxidaceae & Nilappana & $\mathrm{H}$ & $\begin{array}{l}\text { June - } \\
\text { December }\end{array}$ & $\begin{array}{l}\text { Tuberous roots used in skin } \\
\text { troubles, demulcent, diuretic, tonic. } \\
\text { Useful in leucorrhoea, urinary } \\
\text { diseases, piles, jaundice, asthma, } \\
\text { diarrhoea, gonorrhoea, itch and skin } \\
\text { diseases. }\end{array}$ \\
\hline 19 & 053 & $\begin{array}{l}\text { Delonix regia (Boj. ex } \\
\text { Hook) Rafin. }\end{array}$ & Caesalpiniaceae & Poomaram & $\mathrm{T}$ & $\begin{array}{l}\text { February - } \\
\text { July }\end{array}$ & $\begin{array}{l}\text { Leaves are used for diseases of } \\
\text { vata, constipation, inflammations, } \\
\text { arthritis, hemiplegia and } \\
\text { dysmenorrhoea. }\end{array}$ \\
\hline 20 & 055 & Dioscorea bulbifera L. & Dioscoreaceae & Kattukachil & $\mathrm{C}$ & $\begin{array}{l}\text { September - } \\
\text { October }\end{array}$ & $\begin{array}{l}\text { Tubers used for ulcers, piles, } \\
\text { leprosy, worm infestation, cardiac } \\
\text { diseases, polyuria, urinary calculi, } \\
\text { aphrodisiac, rejuvenator, dysentery } \\
\text { and syphilis. }\end{array}$ \\
\hline 21 & 052 & $\begin{array}{l}\text { Diploclisia glaucescens } \\
\text { (Blume) Diels }\end{array}$ & Menispermaceae & Vattavalli & $\mathrm{C}$ & $\begin{array}{l}\text { March - } \\
\text { August }\end{array}$ & $\begin{array}{l}\text { Leaf powder with milk given in } \\
\text { biliousness, gonorrhoea and } \\
\text { syphilis. }\end{array}$ \\
\hline
\end{tabular}




\begin{tabular}{|c|c|c|c|c|c|c|c|}
\hline 22 & 266 & Ficus benghalensis L. & Moraceae & Peraal & $\mathrm{T}$ & $\begin{array}{l}\text { May - } \\
\text { August }\end{array}$ & $\begin{array}{l}\text { Bark is used in skin diseases, cures } \\
\text { dysentery, diarrhoea, leucorrhoea, } \\
\text { nervous disorders and reduces } \\
\text { blood sugar in diabetes. }\end{array}$ \\
\hline 23 & 049 & $\begin{array}{l}\text { Gliricidia sepium (Jacq.) } \\
\text { Kunth ex Walp. }\end{array}$ & Fabaceae & Seemakonna & $\mathrm{T}$ & $\begin{array}{l}\text { March - } \\
\text { May }\end{array}$ & Used for headache, cold and cough. \\
\hline 24 & 050 & Gloriosa superba L. & Liliaceae & Menthonni & $\mathrm{C}$ & $\begin{array}{l}\text { July - } \\
\text { December }\end{array}$ & $\begin{array}{l}\text { Tubers used for swelling, piles, } \\
\text { oedema, leprosy, cronic ulcers, } \\
\text { colic pain in the bladder, itching, } \\
\text { antidote against cobra poison; easy } \\
\text { and quick expulsion of the placenta } \\
\text { after delivery. }\end{array}$ \\
\hline 25 & 229 & $\begin{array}{l}\text { Glycosmis pentaphylla } \\
\text { (Retz.) DC. }\end{array}$ & Rutaceae & Panal & $\mathrm{S}$ & $\begin{array}{l}\text { September - } \\
\text { April }\end{array}$ & $\begin{array}{l}\text { Leaf juice used in fever and liver } \\
\text { complaints and as a vermifuge. } \\
\text { Leaves considered good antidote } \\
\text { for inflammations, fever, } \\
\text { helminthiasis, cough, bronchitis, } \\
\text { rheumatism, jaundice, anaemia, } \\
\text { hepatopathy and skin diseases. }\end{array}$ \\
\hline 26 & 123 & $\begin{array}{l}\text { Grewia nervosa (Lour.) } \\
\text { Panigrahi }\end{array}$ & Tiliaceae & Kotta & $\mathrm{S}$ & $\begin{array}{l}\text { August - } \\
\text { April }\end{array}$ & $\begin{array}{l}\text { The plant is used for indigestion, } \\
\text { eczema and itch, typhoid fever, } \\
\text { dysentery and syphilitic ulceration } \\
\text { of the mouth. }\end{array}$ \\
\hline 27 & 203 & $\begin{array}{l}\text { Hemidesmus indicus (L.) } \\
\text { R.Br. }\end{array}$ & Periplocaceae & Nannari & $\mathrm{C}$ & $\begin{array}{l}\text { August - } \\
\text { December }\end{array}$ & $\begin{array}{l}\text { Roots used for cooling and blood } \\
\text { purifying action, dyspepsia, } \\
\text { dysentery, cough, bronchitis, } \\
\text { uterine haemorrhage, wounds, } \\
\text { leprosy, blood diseases, anaemia, } \\
\text { jaundice, fever, thirst, vomiting, } \\
\text { rheumatism and skin diseases. }\end{array}$ \\
\hline 28 & 253 & $\begin{array}{l}\text { Jasminum sambac (L.) } \\
\text { Ait. }\end{array}$ & Oleaceae & Mulla & $\mathrm{C}$ & $\begin{array}{l}\text { Throughout } \\
\text { the year }\end{array}$ & $\begin{array}{l}\text { Roots, leaves and flowers used in } \\
\text { ophthalmopathy, pruritus, } \\
\text { cephalalgia, otopathy, skin diseases, } \\
\text { haemorrhage, wounds, ulcers, fever, } \\
\text { itching, headache, vomiting, } \\
\text { hiccough and galactorrhea. }\end{array}$ \\
\hline 29 & 048 & $\begin{array}{l}\text { Leea indica (Burm.f.) } \\
\text { Merr. }\end{array}$ & Leeaceae & Chorianthali & $\mathrm{S}$ & $\begin{array}{l}\text { March - } \\
\text { August }\end{array}$ & $\begin{array}{l}\text { Roots used in diarrhoea, dysentery, } \\
\text { hyperdipsia, ulcer and skin } \\
\text { diseases. }\end{array}$ \\
\hline 30 & 095 & $\begin{array}{l}\text { Macaranga peltata } \\
\text { (Roxb.) Muell.-Arg. }\end{array}$ & Euphorbiaceae & Vatta & $\mathrm{T}$ & $\begin{array}{l}\text { January - } \\
\text { February }\end{array}$ & $\begin{array}{l}\text { Decoction of leaves and bark used } \\
\text { as vulnerary. Gum used for } \\
\text { venereal sores. }\end{array}$ \\
\hline 31 & 211 & $\begin{array}{l}\text { Mallotus philippensis } \\
\text { (Lam.) Muell.-Arg. }\end{array}$ & Euphorbiaceae & Sindooram & $\mathrm{T}$ & $\begin{array}{l}\text { October - } \\
\text { March }\end{array}$ & $\begin{array}{l}\text { Used against tapeworms, abdominal } \\
\text { disorders, haemopathy, calculus, } \\
\text { flatulence, leprosy, skin diseases } \\
\text { and ringworm. }\end{array}$ \\
\hline
\end{tabular}




\begin{tabular}{|c|c|c|c|c|c|c|c|}
\hline 32 & 206 & $\begin{array}{l}\text { Merremia vitifolia } \\
\text { (Burm. f.) Hall. f. }\end{array}$ & Convolvulaceae & $\begin{array}{l}\text { Manjavayar- } \\
\text { avalli }\end{array}$ & $\mathrm{C}$ & $\begin{array}{l}\text { November - } \\
\text { February }\end{array}$ & $\begin{array}{l}\text { Whole plant used for strangury and } \\
\text { urethral discharges. Root eaten by } \\
\text { tribals as a stomachic. }\end{array}$ \\
\hline 33 & 189 & $\begin{array}{l}\text { Morinda pubescens J.E. } \\
\text { Smith }\end{array}$ & Rubiaceae & Manjapavitta & $\mathrm{T}$ & $\begin{array}{l}\text { March - } \\
\text { June }\end{array}$ & $\begin{array}{l}\text { It is used for eczema, fever, ulcers, } \\
\text { glandular swellings and digestive } \\
\text { disorders especially in children. }\end{array}$ \\
\hline 34 & 025 & $\begin{array}{l}\text { Mukia maderaspatana } \\
\text { (L.) Roem. }\end{array}$ & Cucurbitaceae & $\begin{array}{l}\text { Kasappuch- } \\
\text { edi }\end{array}$ & $\mathrm{C}$ & $\begin{array}{l}\text { Throughout } \\
\text { the year }\end{array}$ & $\begin{array}{l}\text { Whole plant used for burning } \\
\text { sensation, flatulence, constipation, } \\
\text { ulcers, cough, neuralgia, odontalgia } \\
\text { and vertigo. Fruits used for dysuria, } \\
\text { piles, polyuria, tuberculosis and } \\
\text { alleviating pitta. }\end{array}$ \\
\hline 35 & 085 & $\begin{array}{l}\text { Murraya koenigii (L.) } \\
\text { Spreng. }\end{array}$ & Rutaceae & Kariveppu & $\mathrm{S}$ & $\begin{array}{l}\text { March - } \\
\text { July }\end{array}$ & $\begin{array}{l}\text { Juice of roots used for relief from } \\
\text { renal pains. Leaves improves voice, } \\
\text { stimulates digestion and destroys } \\
\text { concocted poisons in the system; } \\
\text { skin diseases, worm troubles and } \\
\text { neurosis. }\end{array}$ \\
\hline 36 & 190 & $\begin{array}{l}\text { Naravelia zeylanica } \\
\text { (L.) DC. }\end{array}$ & Ranunculaceae & Vathamkodi & $\mathrm{C}$ & $\begin{array}{l}\text { October - } \\
\text { April }\end{array}$ & $\begin{array}{l}\text { Whole plant is used in } \\
\text { helminthiasis, dermatopathy, } \\
\text { leprosy, rheumatalgia, odontalgia, } \\
\text { cephalalgia, inflammations, wounds } \\
\text { and ulcers. }\end{array}$ \\
\hline 37 & 282 & $\begin{array}{l}\text { Pedilanthus tithymaloides } \\
\text { (L.) Poir. }\end{array}$ & Euphorbiaceae & Zigzag plant & $S$ & $\begin{array}{l}\text { April - } \\
\text { August }\end{array}$ & Wound healing property. \\
\hline 38 & 045 & $\begin{array}{l}\text { Phyllanthus amarus } \\
\text { Schum. \& Thonn. }\end{array}$ & Euphorbiaceae & Keezharnalli & $\mathrm{H}$ & $\begin{array}{l}\text { July - } \\
\text { October }\end{array}$ & $\begin{array}{l}\text { The plant is used for flu, dropsy, } \\
\text { diabetes, jaundice, asthma, } \\
\text { bronchial infections, diseases of the } \\
\text { liver, stomach, genito-urinary } \\
\text { system, liver and kidney. The plant } \\
\text { is reported to show antiviral activity } \\
\text { against hepatitis B-virus and related } \\
\text { hepadna virus. }\end{array}$ \\
\hline 39 & 160 & $\begin{array}{l}\text { Phyllanthus reticulatus } \\
\text { Poir. in Lam. }\end{array}$ & Euphorbiaceae & Neeroli & $S$ & $\begin{array}{l}\text { August - } \\
\text { December }\end{array}$ & $\begin{array}{l}\text { Bark used in rheumatism, dysentery } \\
\text { and venereal diseases. Leaves used } \\
\text { for burning sensation, gastropathy, } \\
\text { sores, burns, skin eruptions and } \\
\text { obesity. Fruits are used in dressing } \\
\text { syphilitic sores. }\end{array}$ \\
\hline 40 & 307 & Physalis angulata L. & Solanaceae & $\begin{array}{l}\text { Njottanjod- } \\
\text { ian }\end{array}$ & $\mathrm{H}$ & $\begin{array}{l}\text { July - } \\
\text { December }\end{array}$ & $\begin{array}{l}\text { Roots boiled in water suppresses } \\
\text { diabetes, heal sores of mouth. } \\
\text { Whole plant used for burning } \\
\text { sensation, ulcers, gastropathy, } \\
\text { cough and bronchitis. }\end{array}$ \\
\hline 41 & 088 & Piper longum L. & Piperaceae & Thippali & $\mathrm{C}$ & $\begin{array}{l}\text { August - } \\
\text { January }\end{array}$ & $\begin{array}{l}\text { Roots and fruits are used for } \\
\text { improve intellect memory power } \\
\text { and regain health by dispelling } \\
\text { diseases. It also cures cough, } \\
\text { asthma, indigestion, worm troubles, } \\
\text { anaemia and chronic fever. }\end{array}$ \\
\hline
\end{tabular}




\begin{tabular}{|c|c|c|c|c|c|c|c|}
\hline 42 & 009 & Pothos scandens L. & Araceae & Paruvakodi & $\mathrm{C}$ & $\begin{array}{l}\text { October - } \\
\text { November }\end{array}$ & $\begin{array}{l}\text { Whole plant is used in skin } \\
\text { diseases, boils, swellings, wounds, } \\
\text { ulcers, dropsy, menorrhagia, } \\
\text { vomiting, flatulence, strangury and } \\
\text { burning sensation. }\end{array}$ \\
\hline 43 & 042 & $\begin{array}{l}\text { Putranjiva roxburghii } \\
\text { Wall. }\end{array}$ & Euphorbiaceae & Poothilanji & $\mathrm{T}$ & $\begin{array}{l}\text { March - } \\
\text { August }\end{array}$ & $\begin{array}{l}\text { Leaves and seeds are used for } \\
\text { burning sensation, thrist, } \\
\text { stomatopathy, opthalmopathy, } \\
\text { constipation, elephantiasis and } \\
\text { habitual abortion. }\end{array}$ \\
\hline 44 & 181 & $\begin{array}{l}\text { Schleichera oleosa } \\
\text { (Lour.) Oken }\end{array}$ & Sapindaceae & Poovam & $\mathrm{T}$ & $\begin{array}{l}\text { March - } \\
\text { June }\end{array}$ & $\begin{array}{l}\text { Bark useful in curing ulcers, } \\
\text { malaria and inflammations. Seed } \\
\text { oil used in leprosy, dermatopathy, } \\
\text { boils, ulcers, blood disorders, } \\
\text { intermittent fever, snakebite and } \\
\text { burns. }\end{array}$ \\
\hline 45 & 030 & Senna tora (L.) Roxb. & Caesalpiniaceae & Thakara & $\mathrm{H}$ & $\begin{array}{l}\text { August - } \\
\text { December }\end{array}$ & $\begin{array}{l}\text { Leaves and seeds used in ringworm, } \\
\text { leprosy, skin diseases, constipation, } \\
\text { abdominal disorders, obesity, } \\
\text { flatulence helminthiasis and } \\
\text { constipation. }\end{array}$ \\
\hline 46 & 108 & Sida acuta Burm. f. & Malvaceae & Kurumthotti & $\mathrm{S}$ & $\begin{array}{l}\text { August - } \\
\text { October }\end{array}$ & $\begin{array}{l}\text { Used for uropathy, arthritis, } \\
\text { leucorrhoea, gonorrhoea, diarrhoea } \\
\text { and to promote strength. }\end{array}$ \\
\hline 47 & 031 & Sida cordifolia L. & Malvaceae & $\begin{array}{l}\text { Anakurumt- } \\
\text { hotti }\end{array}$ & $\mathrm{S}$ & $\begin{array}{l}\text { Throughout } \\
\text { the year }\end{array}$ & $\begin{array}{l}\text { Roots given in urinary troubles, } \\
\text { stranguary and haematuria, used in } \\
\text { combination with asafoetida in } \\
\text { hemiplegia, sciatica and facial } \\
\text { paralysis (with rock salt). Powdered } \\
\text { roots are given with milk in } \\
\text { leucorrhoea and frequent } \\
\text { micturition. Leaves demulcent and } \\
\text { febrifuge; also used in dysentery. } \\
\text { Roots are used for rheumatism, } \\
\text { headache, neurological disorders, } \\
\text { tuberculosis and ophthalmia. }\end{array}$ \\
\hline 48 & 090 & Streblus asper Lour. & Moraceae & Paruka & $\mathrm{T}$ & $\begin{array}{l}\text { January - } \\
\text { October }\end{array}$ & $\begin{array}{l}\text { Bark, roots and seeds are used for } \\
\text { sinusitis, inflammations, } \\
\text { elephantiasis, haemorrhages, cough, } \\
\text { bronchitis, foul ulcers, diarrhoea, } \\
\text { dysentery, fever, swellings, } \\
\text { neuralgia and haemorrhages. }\end{array}$ \\
\hline 49 & 039 & Strychnos nux-vomica L. & Loganiaceae & Kanjiram & $\mathrm{C}$ & $\begin{array}{l}\text { March - } \\
\text { December }\end{array}$ & $\begin{array}{l}\text { Seeds are useful in intermittent } \\
\text { fevers, dyspepsia, chronic } \\
\text { dysentery, paralytic and neuralgic } \\
\text { affections, insomnia, chronic } \\
\text { rheumatism, colic, impotence, heart } \\
\text { disease, spermatorrhoea and skin } \\
\text { diseases. }\end{array}$ \\
\hline
\end{tabular}




\begin{tabular}{|c|c|c|c|c|c|c|c|}
\hline 50 & 308 & $\begin{array}{l}\text { Terminalia paniculata } \\
\text { Roth }\end{array}$ & Combretaceae & Maruthu & $\mathrm{T}$ & $\begin{array}{l}\text { August - } \\
\text { February }\end{array}$ & Bark is a cardio tonic and diuretic. \\
\hline 51 & 165 & $\begin{array}{l}\text { Tiliacora acuminata } \\
\text { (Poir.) Miers ex Hook. f. } \\
\text { \& Thomas. }\end{array}$ & Menispermaceae & Vallikanjiram & $\mathrm{C}$ & $\begin{array}{l}\text { April- } \\
\text { December }\end{array}$ & $\begin{array}{l}\text { Roots are used as an antidote to } \\
\text { snake poison. }\end{array}$ \\
\hline 52 & 222 & $\begin{array}{l}\text { Tinospora cordifolia } \\
\text { (Willd.) Miers. }\end{array}$ & Menispermaceae & Chitamruthu & $\mathrm{C}$ & $\begin{array}{l}\text { January - } \\
\text { June }\end{array}$ & $\begin{array}{l}\text { Stems used in fever, jaundice, } \\
\text { thirst, burning sensation, diabetes, } \\
\text { piles, skin ailments, respiratory } \\
\text { disorders, neurological disorders } \\
\text { and rheumatism. }\end{array}$ \\
\hline 53 & 037 & $\begin{array}{l}\text { Tinospora sinensis } \\
\text { (Lour.) Merr. }\end{array}$ & Menispermaceae & Katamruthu & $\mathrm{C}$ & $\begin{array}{l}\text { February - } \\
\text { June }\end{array}$ & $\begin{array}{l}\text { Stems used for treatment of piles } \\
\text { and ulcerated wounds, liver } \\
\text { complaints, chronic rheumatism } \\
\text { and also as muscle relaxant. }\end{array}$ \\
\hline 54 & 134 & $\begin{array}{l}\text { Triumfetta rhomboidea } \\
\text { Jacq. }\end{array}$ & Tiliaceae & Oorpam & $\mathrm{S}$ & $\begin{array}{l}\text { August - } \\
\text { January }\end{array}$ & $\begin{array}{l}\text { Roots used in dysentery, intestinal } \\
\text { ulcers and their hot infusion hasten } \\
\text { parturition. Bark and leaves used in } \\
\text { diarrhoea. Leaves and flowers used } \\
\text { in leprosy. }\end{array}$ \\
\hline 55 & 138 & Urena lobata $\mathrm{L}$. & Malvaceae & Uthiram & $\mathrm{S}$ & $\begin{array}{l}\text { August - } \\
\text { December }\end{array}$ & $\begin{array}{l}\text { Decoction of stem and roots are } \\
\text { used in flatulent colic. Flowers are } \\
\text { used for cough and sore throat. }\end{array}$ \\
\hline 56 & 035 & $\begin{array}{l}\text { Vernonia cinerea }(\mathrm{L} .) \\
\text { Less. }\end{array}$ & Asteraceae & $\begin{array}{l}\text { Puvankuru- } \\
\text { nal }\end{array}$ & $\mathrm{H}$ & $\begin{array}{l}\text { Throughout } \\
\text { the year }\end{array}$ & $\begin{array}{l}\text { Useful combination with quinine } \\
\text { against malaria. Roots used for } \\
\text { fever, dysuria, leucorrhoea, } \\
\text { excessive bleeding, chronic skin } \\
\text { diseases, bladder stones, piles, } \\
\text { worms and haematological } \\
\text { disorders. The plant juice is good } \\
\text { for eyes. }\end{array}$ \\
\hline 57 & 184 & $\begin{array}{l}\text { Zanthoxylum rhetsa } \\
\text { (Roxb.) DC. }\end{array}$ & Rutaceae & Mullilam & $\mathrm{T}$ & $\begin{array}{l}\text { March - } \\
\text { November }\end{array}$ & $\begin{array}{l}\text { Bark and fruits used in dyspepsia, } \\
\text { asthma, bronchitis, heart diseases, } \\
\text { toothache, diseases of eye and ear, } \\
\text { worm infestation, leprosy, diseases } \\
\text { of head and rheumatism. Seeds are } \\
\text { used in cholera. Thorns are used in } \\
\text { treating pimples. }\end{array}$ \\
\hline
\end{tabular}

\section{DISCUSSION}

As the study area (Chithalikavu) is near to an all-weather road, passing through its Southern side and there is no compound wall around the sacred grove; various anthropological disturbances are common and these affect the normal growth of natural flora. Floral diversity studies show that regional diversity is well represented in grove system, larger groves often have the relic species of the region and there are frequent changes in floral composition due to various external influences (Khumbongmayum et al. 2006, Rao et al. 1990). Regeneration of tree species in this area was very poor because of zoo-anthropogenic activities, which also causes the soil erosion and finally decreasing the soil fertility.

Invasive species Chromolaena odorata (L.) King \& Robins. and Carica papaya L. are present inside this grove. Overgrowth of these plants is considered as a threat to the other vegetation of the sacred groves. 
Chromolaena odorata is most widely spreading and encroaching species of the similar areas (Cronk \& Fuller 1995, Richardson \& Rejmánek 2011, Mandal \& Joshi 2014a).

Table 2. Phytosocioloical analysis of major tree species in Chithalikavu.

\begin{tabular}{llrrrrrrr}
\hline S.N. & \multicolumn{1}{c}{ Name of species } & D $($ No./ha) & Ab & F & RD & RF & RBA & IVI \\
\hline 1 & Caryota urens L. & 37.5 & 1.5 & 1.0 & 0.083 & 0.182 & 0.004 & 0.269 \\
2 & Delonix regia (Boj. ex Hook) Rafin. & 12.5 & 1.0 & 0.5 & 0.028 & 0.091 & 0.003 & 0.121 \\
3 & Ficus benghalensis L. & 12.5 & 1.0 & 0.5 & 0.028 & 0.091 & 0.515 & 0.634 \\
4 & Putranjiva roxburghii Wall. & 12.5 & 1.0 & 0.5 & 0.028 & 0.091 & 0.001 & 0.119 \\
5 & Schleichera oleosa (Lour.) Oken & 12.5 & 1.0 & 0.5 & 0.028 & 0.091 & 0.190 & 0.309 \\
6 & Strychnosnux-vomica L. & 325.0 & 13.0 & 1.0 & 0.722 & 0.182 & 0.181 & 1.085 \\
7 & Terminalia paniculata Roth & 25 & 1.0 & 1.0 & 0.056 & 0.182 & 0.101 & 0.338 \\
8 & Zanthoxylum rhetsa (Roxb.) DC. & 12.5 & 1.0 & 0.5 & 0.028 & 0.091 & 0.007 & 0.125 \\
\hline
\end{tabular}

Note: D- Density, Ab- Abundance, F- Frequency, RD- Relative density, RF- Relative frequency, RBA- Relative basal area, IVI- Importance value index.

\section{CONCLUSION}

Groves are important for conservation of biological diversity. Its values and functions can be maintained through effective conservation and management. Almost all species of flora is medicinal inside the grove and used in various systems of medicine. This study shows due to diverse threats the floristic diversity reaches near to its minimum level. Therefore increase in demand to conservation to maintain and increase biodiversity. Local people must become aware of direct benefits of groves and they can learn more about the functions. The above facts focus the aspects of conservation of groves, facing great danger of existence due to loss of sanctity values.

\section{ACKNOWLEDGEMENTS}

Authors are grateful to Sri. D. Jayaprasad, Principal and Dr. G. Jayakrishnan, Department of Botany, Sree Krishna College, Guruvayur for providing valuable suggestions for the work. Authors acknowledge the family members of these sacred groves for granting permission to conduct the study and providing information about the groves.

\section{REFERENCES}

Bajpai O, Kushwaha AK, Srivastava AK, Pandey J \& Chaudhary LB (2015) Phytosociological status of a monotypic genus Indopiptadenia: a near threatened tree from the Terai-Bhabar Region of Central Himalaya. Research Journal of Forestry 9(2): 35-47.

Cronk QCB \& Fuller JI (1995) Plant invaders: the threat to natural ecosystems. Chapman and hall, London.

Deepa MR, Sheema Dharmapal P \& Udayan PS (2016) Floristic diversities and medicinal importance of selected sacred groves in Thrissur district, Kerala. Tropical Plant Research 3(1): 230-242.

Dregne HE (1987) Soil erosion: cause and effect. Land use policy 4(4): 412-418.

Gaikwad SS, Paralikar SN, Chavan V \& Krishnan S (2004) Digitizing Indian sacred groves - An information model for web interfaced multimedia database. In: Vinya G, Sane H and Ranade SS (eds) Focus on Sacred Groves and Ethnobotany. Prisam Publications, Mumbai, India, pp.123-128.

Gamble JS \& Fischer CEC (1915-1936) The Flora of the Presidency of Madras. Parts 1-11 (parts 1-7 by Gamble and 8-11 by Fischer), Vols. 1-3. Adlard \& Sons Ltd., London.

Jain SK \& Rao RR (1977) A Handbook of Field and Herbarium Methods. Today and Tomorrow, New Delhi.

Khumbongmayum AD, Khan ML \& Tripathi RS (2006) Biodiversity conservation in sacred groves of Manipur, northeast India: population structure and regeneration status of woody species. Biodiversity and Conservation 15: 2439-2456.

Malhotra KC, Gokhale Y \& Chatterjee S (2001) Cultural and Ecological Dimensions of Sacred Groves in India. Indian National Science Academy, New Delhi and Indira Gandhi Rashtriya Manav Sangrahalaya, Bhopal.

Mandal G \& Joshi SP (2014a) Quantitative vegetation dynamics and invasion success of Lantana camara from the tropical forests of doon valley. International Journal of conservation science 5(4): 511-526.

Nair NC \& Mohanan CN (1981) On the rediscovery of four threatened species from the sacred groves of Kerala. Journal of Economic and Taxonomic Botany 2: 233-235.

Parthasrathy N \& Karthikeyan R (1997) Biodiversity and population density of woody species in a tropical evergreen forest in Courtallum reserve forest, Western Ghats India. Tropical ecology 38: 297-306.

www.tropicalplantresearch.com 
Pascal JP \& Pelissier R (1996) Structure and floristic composition of a tropical evergreen forest in southwest India. Journal of Tropical Ecology 12: 191-214.

Poffenberger M, Mc Gran B, Ravindranath NH \& Gadgil M (1992) Field methods Manual volume 1. Diagnostic tool for supporting joint forest Management System. Prepared for the joint forest Management Support programme.

Pushpangadan P, Krishnan PN \& Rajendraprasad M (1998) The life form spectrum of sacred groves - a functional tool to analyze the vegetation. Tropical Ecology 39(2): 211.

Rao P, Barik SK, Pandey HN \& Tripathi RS (1990) Community composition and tree population structure in a sub-tropical broad-leaved forest along a disturbance gradient. Vegetation 88: 151-162.

Ravikumar K, Ved DK Assisted by Vijaya Sankar R \& Udayan PS (2000) 100 Red-Listed Medicinal Plants of Conservation Concern in Southern India. Foundation for Revitalisation of Local Health Traditions (FRLHT), 50, MSH Lay out, $2^{\text {nd }}$ stage, $3^{\text {rd }}$ main, Anand Nagar, Banglore, India.

Richardson DM \& Rejmánek M (2011) Trees and shrubs as invasive alien species - a global review. Diversity and Distribution 17: 788-809.

Sasidharan N \& Sivarajan VV (1996) Flowering Plants of Thrissur Forest (Western Ghats, Kerala, India). Scientific Publishers, Jodhpur.

Shailaja R \& Sudha P (2001) Biomass Studies; Field methods for monitoring Biomass. Oxford \& IBH Publishing Co. Pvt. Ltd. New Delhi.

Venkatachalam, Kalaiselvi ST \& Ratha Krishnan P (2005) Sacred Groves - Conservation is Imperative. Institute of Forest Genetics and Tree Breeding, PB 1061, R.S.Puram, Coimbatore 641002, India, pp. 93-96. 